\title{
Impact of Carbamide Peroxide whitening agent on dentinal collagen
}

O. Redha ${ }^{1}$, A. Strange ${ }^{2}$, A. Maeva ${ }^{2}$ R. Sambrook ${ }^{1}$, Nicky Mordan², A. McDonald ${ }^{1}$ and L. Bozec ${ }^{2,3^{*}}$

${ }^{1}$ Division of Prosthodontics, UCL Eastman Dental Institute, University College London, London, UK

${ }^{2}$ Division of Biomaterials and Tissue Engineering, UCL Eastman Dental Institute, University College London, London, UK

${ }^{3}$ Faculty of Dentistry, University of Toronto, Toronto, Canada

Abstract word count: 288

Total word count (Abstract to acknowledgements): 3313

Number of figures: 5

Number of references: 40 (13 from the last 5 years - 30\%)

Keywords: carbamide peroxide, Raman Spectroscopy, dentin, collagen, scanning electron microscopy, whitening

\section{${ }^{*}$ Corresponding author:}

Laurent Bozec DUT, BSc(Hons), PhD

Professor (Associate) in Nanometrology \& Dental Sciences

Faculty of Dentistry | University of Toronto

124 Edward Street, Room 459 | Toronto, ON M5G 1 G6

Office:+1(416).864.8226 |Cell:+1(437)929. 6566

Skype: I.bozec | Twitter: L_Bozec

Email: I.bozec@utoronto.ca

https://www.dentistry.utoronto.ca/faculty-profiles/laurent-bozec 


\section{Abstract :}

Carbamide peroxide (CP) is widely used as a tooth whitening agent in selfadministered tooth bleaching products. In this study, the effects of $5 \%$ and $10 \% \mathrm{CP}$ on dentinal collagen structure and chemical properties were evaluated in-vitro. Thirtyfive (35) intact teeth were exposed to two whitening protocols ( 2 or 4 hours daily) with either $5 \%$ or $10 \%$ CP gel for 1 -week. Shade changes before and after the whitening protocol were captured colourimetrically using a spectroshade. Collagen scaffold models and demineralised dentine disc samples were prepared and exposed to CP droplets $(5 \%$ or $10 \%)$. Structural changes were investigated using Electron Microscopy. Finally, mineralised dentine disc samples were prepared post-bleaching to assess chemical changes resulting from $\mathrm{CP}$ exposure in dentinal collagen using Raman Spectroscopy.

Results showed a difference in tooth shade when exposed to $5 \%$ and $10 \% \mathrm{CP}$ whitening protocols, with a significant $(p \leq 0.01)$ greater change reported for the $10 \% \mathrm{CP} / 4 \mathrm{hrs}$ group. Imaging of the collagen scaffold model following exposure to $\mathrm{CP}$ showed a gelatinisation process indicating that the free-radical by-products from CP are able to disrupt the quaternary structure of non-crosslinked collagen. The most significant damage on the collagen scaffold was seen for the $10 \%$ CP exposure for 4 hours. Imaging of the demineralised discs displays the same glassy amorphous layer appearance as found in the collagen scaffold. Raman spectra of the mineralised dentine discs showed a significant decrease $(p \leq 0.01)$ in the integrated area of amide I and amide III values in the four test groups following CP application. Amide I was more affected as both the exposure time and concentration of CP increased.

In spite of the claimed safety of whitening agents, this in-vitro study concludes that even low concentrations of $\mathrm{CP}$, results in a deleterious change in dentinal collagen. 


\section{Introduction:}

With the great emphasis on dental aesthetics, the popularity of home dental whitening has increased extensively in recent years. Although the first report on vital tooth whitening dates back to 1848 with the use of chloride of lime (Dwinelle 1850), the contemporary demand for whiter teeth has driven the development of numerous methods and products available. Amongst these is Carbamide Peroxide (CP). The use of $\mathrm{CP}$ for home teeth whitening was first reported by Haywood and Haymann in 1989 (Haywood and Heymann 1989) as a $10 \%$ gel delivered via a custom fabricated appliance. Carbamide peroxide $\left(\mathrm{CO}\left(\mathrm{NH}_{2}\right) \mathrm{H}_{2} \mathrm{O}_{2}\right)$ is an organic white crystalline compound formed by urea and hydrogen peroxide (Toledano et al. 2011). It is also known as urea peroxide, urea hydrogen peroxide (UHP), ortizon and per carbamide.

On contact with teeth, CP breaks down into hydrogen peroxide (HP) and urea, with the former being the active ingredient. The mechanism of tooth whitening is dependent on the penetration of the released peroxide free radicals through the enamel and into the dentine (Elfallah et al. 2015; Hanks et al. 1993; Kwon and Wertz 2015). It is understood that the free radicals subsequently break down the dentine chromogenic molecules into smaller structures or alter their optical properties leading to the removal of stain appearance. Both the initial concentration of the peroxide and the duration of application are the two main factors in determining the overall tooth whitening efficacy of peroxide-containing products (Joiner 2006). Indeed, the higher the concentration and the longer the application time, the whiter the outcome. However, besides obtaining the desired change in the tooth's colour, it would seem appropriate to evaluate the impact of these concentrations and application times on the tooth 
structure (Soares et al. 2014).

One of the main components of tooth structure is dentine. Dentine itself is a hierarchical composite, consisting of $\sim 70 \mathrm{wt} \%$ apatite, with the remainder being mostly collagen and water (Goldberg et al. 2011). Structurally, dentine can be described as a composite of nanocrystalline hydroxyapatite $(\mathrm{HA})$ platelets ( $\sim \mathrm{nm}$ thick), embedded in a softer highly-organised collagen-fibril scaffold. At the mesoscale, these mineralised collagen fibrils are organized radially about hollow or protein filled tubules (1-2.5 $\mu \mathrm{m}$ in diameter) that radiate from the pulp cavity to the dentin surface. This network of tubules provides routes for the transport of fluids, ions, and molecules into the dentinal material. The physical properties of dentine are conferred by the composite arrangement of $\mathrm{HA}$ and collagen. Subsequently it is prudent to evaluate the impact of the aforementioned tooth whitening procedures on the dentine structure (Haywood 2006; Joiner 2006).

The aim of this in-vitro study was to determine the effects of CP used in tooth whitening and explore the influence of concentration and exposure time of the peroxide agent on dentinal collagen structure. Therefore, the null-hypothesis investigated was "Carbamide peroxide whitening agent $(5 \%$ or $10 \%)$ does not affect the structure of dentine collagen"

\section{Materials and Methods}

\section{Carbamide Peroxide gel}

Tooth whitening kits were sourced directly from the manufacturer (undisclosed), in the form of a ready to use gel. Two CP gels were used with a concentration of $5 \%$ and $10 \%$ respectively. The chemical composition of the CP gel was: Water (Aqua), 
Glycerine, Carbomer (Sodium salt) Hydrogen Peroxide, Sodium Tripolyphosphate, Urea, Potassium Nitrate, Sodium Fluoride and Aroma.

\section{Non-cross linked Collagen scaffold model}

Collagen (First Link, Birmingham, West Midlands, UK) scaffolds were prepared using a developed standardised protocol (Cheema and Brown 2013). Subsequently, the scaffolds were physisorbed onto glass microscope slides before being exposed to $5 \%$ and $10 \%$ CP for four hours.

\section{Collection of tooth samples}

A total of 38 intact human premolars and third molars extracted for orthodontic reasons (age group 14-21), were collected for this study under ethical approval (study number 1703) from UCL Eastman BioBank. Informed patient consent was sought from each tooth used in this study. Following extraction, the teeth were initially stored in a $70 \%$ ethanol solution for up to 5 days at room temperature before being debrided from remaining soft tissues and finally stored in a $0.1 \%$ thymol solution at $4^{\circ} \mathrm{C}$ until required for the study (storage did not exceed 2 months). The sample size was calculated in G* Power 3.1.9.2 using power of $80 \%(0.8)$ and $\alpha$ error probability 0.05 by estimating the required effect size (from Raman spectra) in a pilot study. The 35 teeth collected were split randomly in 5 treatment groups of 7 samples (control; $5 \% \mathrm{CP} / 2 \mathrm{hrs}$; 5\%CP/4hrs; 10\%CP/2hrs and 10\% CP/4hrs).

\section{Preparation of tooth samples}

4.1 Demineralised dentine discs

Three (3) teeth were sectioned transversely at the mid-coronal level to obtain $3 \mathrm{~mm}$ thick disc samples using a diamond microtome (Struers, Accutom-50, Struers Ltd., Solihull, West Midlands, UK). To expose the dentinal collagen, these discs were immersed in $37 \%$ phosphoric acid in a sonic bath for up to 15 seconds (Han et al. 
2017) followed by 2 minutes in distilled water then air-drying. The level of demineralisation was carefully monitored using ATR-FTIR spectroscopy through the ratio of amide I to phosphate peak (data not shown). The ratio of amide I and III bands was also monitored to ensure that the dentinal collagen was not affected by the acid demineralisation process. Then, the discs were exposed to a droplet of either $5 \%$ or $10 \% \mathrm{CP}$ for 4 hours in a delineated area whereas the rest of the surface of the unexposed disc served as control.

\subsection{Mineralised dentine discs}

Thirty-five (35) tooth samples were coated in nail varnish except for a window of $5 \mathrm{x}$ $5 \mathrm{~mm}$ on both the buccal and lingual/palatal surfaces, to be exposed to CP (Figure 1).

a) Tooth whitening protocol

The tooth samples were randomly assigned to 3 groups: control and two groups exposed to $5 \%$ and $10 \% \mathrm{CP}$ respectively. A pilot study comparing placebo gel vs artificial saliva (AS) yielded no significant difference. For clinical relevance, AS was used for the control group. The CP groups were further divided into two sub-groups $(n=7)$ to accommodate for two different exposure durations. Exposed buccal enamel windows for the test groups were treated with CP gel at either $5 \%$ or $10 \%$ concentrations for 2 or 4 hours daily over a 1 -week period. The CP gel was washed off the tooth surface at the end of each exposure using dionised water. The samples were kept at $37^{\circ} \mathrm{C}$ immersed in AS (McKnight-Hanes and Whitford 1992) during the entire whitening protocol.

b) After the whitening protocol

Following the tooth whitening protocol, the teeth were transversely sectioned at the midpoint of the buccal window, which corresponded to the area where CP had been applied, into $3 \mathrm{~mm}$ thick discs using a diamond microtome (Struers, Accutom-50, 
Struers Ltd., Solihull, West Midlands, UK). This allowed exposure of the dentine surface and pulp, buccolingually, for Raman analysis (Figure 1-a). The surface of the discs was then polished by Struers Labopol 5 (Struers Ltd., Solihull, West Midlands, UK) using 2400 grit and diamond polishing paste (Diapro, Struers Ltd., Solihull, West Midlands, UK). A reference scalpel line corresponding to the CP application was created on the polished surfaces to enable identification of 4 different locations across this transverse line buccolingually (Figure 1-b). To remove the smear layer, the discs were ultrasonicated in deionised water for 10 minutes.

\section{Colour measurement}

A Spectroshade (MHT Optic Research AG, Switzerland) was used to determine the shade before and after the tooth whitening protocols, prior to disc preparation. The instrument was calibrated prior to shade measurements. By measuring the $\mathrm{CIE} \mathrm{L}^{*} \mathrm{a}^{*} \mathrm{~b}^{*}$ values, the overall colour difference before and after exposure $(\Delta E)$ was calculated per the following expression (de l'Eclairage 1978; Lima et al. 2018)

$$
\Delta E=\sqrt{\left[\left(\mathrm{L}_{\text {before }}^{*}-\mathrm{L}_{\mathrm{after}}^{*}\right)+\left(\mathrm{a}_{\text {before }}^{*}-\mathrm{a}_{\mathrm{after}}^{*}\right)\left(\mathrm{b}_{\text {before }}^{*}-\mathrm{b}_{\mathrm{after}}^{*}\right)\right]^{2}}
$$

For the spectroshade measurement, each treatment group (5\%CP/2hrs; $5 \% \mathrm{CP} / 4 \mathrm{hrs}$; $10 \% \mathrm{CP} / 2 \mathrm{hrs}$ and $10 \% \mathrm{CP} / 4 \mathrm{hrs}$ ) was assessed pre and post-treatment. The pretreatment assessment acted as a control measurement to evaluate the change of colour.

\section{Raman Spectroscopy}

Raman spectra of the prepared mineralised dentine discs were obtained with a confocal Labram 300 spectrophotometer (Horiba Jobin Yvon, France). The spectra were recorded along the transverse reference line at four locations (Loc1: $1 \mathrm{~mm}$ away from the buccal DEJ, Loc2: $1 \mathrm{~mm}$ away from the buccal pulp, Loc3: $1 \mathrm{~mm}$ away from the lingual pulp wall and Loc4: 1mm away from the lingual DEJ) (Figure 1) . Raman 
spectra of both inter-tubular and peritubular dentine were recorded. For each location, 3 spectra for each type of dentine were recorded before being averaged. All the spectra were baseline corrected and normalised to the carbonate peaks of the control group present at $1072 \mathrm{~cm}^{-1}$. Subsequently, the area of the amide I $\left(1610-1710 \mathrm{~cm}^{-1}\right)$, amide III (1220-1300 $\left.\mathrm{cm}^{-1}\right)$ and phosphate $\left(960 \mathrm{~cm}^{-1}\right)$ bands were integrated. The data was then exported for graphing and analys to OriginPro 9.0 (OriginCorp, USA).

\section{Protocol for SEM}

Following their exposure to $\mathrm{CP}$, the collagen scaffolds and the demineralised dentine discs were fixed in $3 \%$ glutaraldehyde (Agar Scientific, UK) in $0.1 \mathrm{M}$ cacodylate buffer for $24 \mathrm{hr}$. Samples were dehydrated in an ethanol series $(20 \%, 50 \%, 70 \%, 90 \%$ \& $100 \%$ ) each for 15 minutes before air-drying. Samples were mounted on stubs before sputter coated on a Polaron E 5000 (Quorum technology, UK) with gold. These were imaged using a Philips XL30 FEG-SEM (FEI, Eindhoven), with an acceleration voltage of $5 \mathrm{kV}$. Contrast and brightness were optimised for each image.

\section{Statistical Analysis}

One-way ANOVA was conducted to assess the significance in colour change between the groups after exposure to CP followed by post-hoc analysis (Bonferroni test) to evaluate $\Delta \mathrm{E}$ Values. For Raman data, Kruskal-Wallis test was used to assess the spectroscopic impact of CP on dentinal collagen as a function of concentration and exposure time, whereas a Friedman test was used to evaluate the same output for the locations across the buccal and lingual regions respectively. All statistical analyses were performed using SPSS statistical software package (IBM Corp, USA). All spectra were plotted using Origin Pro 9.0 (OriginCorp, USA). 


\section{$\underline{\text { Results and Discussion }}$}

\section{- Colourimetric assessment of the efficacy of CP as a whitening agent}

Home tooth whitening kits have the sole purpose of improving tooth colour. The concentration of peroxide present in $5 \%$ and $10 \% \mathrm{CP}$ is usually relatively low. Although there have been a number of studies showing the efficacy of such peroxide concentrations to lighten the colour of the teeth (De Geus et al. 2018; Meireles et al. 2008; Rao et al. 2016), it was necessary to ensure that the commercial products used in this study performed accordingly. Thus, this initial efficacy study tested the two CP concentrations (5\% and 10\%) applied over a 2 or 4 -hour period for 1 week as recommended by the manufacturer's instructions. The shade $\left(\mathrm{L}^{*}, \mathrm{a}^{*}\right.$, and $\mathrm{b}^{*}$ values) of 28 teeth was recorded before (baseline) and after the whitening protocol (previously described) and the change in $\Delta \mathrm{E}$ calculated. As an example, a sample taken from the $(10 \% \mathrm{CP} / 4 \mathrm{hrs})$ group presented a change in shade: $\Delta \mathrm{E}=-4.1$, whereas a sample taken from the $(5 \% \mathrm{CP} / 4 \mathrm{hrs})$ group presented a change in shade: $\Delta \mathrm{E}=+0.6$ as shown in Figure 2. The overall outcome was a significant difference in the calculated $\Delta \mathrm{E}$ between pre and post CP exposure for the $(10 \% \mathrm{CP} / 4 \mathrm{hrs})$ group $(p \leq 0.01)$ whereas there was no significant difference for the other groups. Similar findings were reported by Suliman in 2006 (Sulieman et al. 2006). This outcome is likely due to the short period of application ( 1 week) in our study. In comparison other studies have reported a change in colour when using $5 \% \mathrm{CP}$ following a three week application period for example (Leonard et al. 1998). Nevertheless, whilst whitening efficacy, in this study, was demonstrated for the $10 \% \mathrm{CP} 4 \mathrm{hrs}$ group only, it is not clear whether the other whitening protocols may induce changes in the dentine components. 


\section{- Effect of CP on the collagen scaffold model and demineralized dentinal}

collagen

The main reason for $\mathrm{CP}$ to be used for tooth whitening is that it decomposes into hydrogen peroxide $\left(\mathrm{H}_{2} \mathrm{O}_{2}\right)$ and urea $\left(\mathrm{CO}\left(\mathrm{NH}_{2}\right)_{2}\right)$. A $10 \%$ CP concentration decomposes into $\sim 3.5 \% \mathrm{HP}$ and $6.5 \%$ urea. Of these two chemical compounds, HP is the most active as it has the potential of releasing free radicals. Coupled with its low-molecular-weight, which enables its diffusion through the organic matrix of the tooth, HP holds the potential of affecting any free-radical sensitive components of the dentine structure, especially proteins (Kou and Takahama 1995; Walsh 2000).

Collagen is the most abundant protein in dentine. In order to evaluate the possible effect of CP on dentinal collagen, the impact of CP on both a non-crosslinked collagen model scaffold (Figure 3-a) as used for Tissue Engineering (Neel et al. 2013) and on demineralised dentinal collagen (Figure 3-b) was investigated. Figure 3-a-i) presents a hydrated collagen scaffold with selected area as either non-exposed to $\mathrm{CP}$, exposed to a CP droplet (5\%CP/4hrs) or exposed to CP droplet $(10 \% \mathrm{CP} / 4 \mathrm{hrs})$. A front of collagen degradation can be observed radiating away from the initial droplet as presented in Figure 3-a-i) for the 2 areas exposed to CP. This front of degradation is reminiscent of a gelatinisation process in which the fibrils are losing their shape/banding periodicity and morphing into a glassy amorphous layer (Bozec and Odlyha 2011) denoted by the star in Figure 3-a. This gelatinisation process becomes more evident at higher magnification (Figure 3-a-iii \&iv) when compared to the nonexposed collagen in Figure 3-a-ii. In these model collagen scaffolds. Close to the gelatinisation front, the fibrils lose their D-banding periodicity and become more swollen in appearance, which is a clear evidence of structural and chemical 
degradation. This result suggests that $\mathrm{CP}$ is able to disrupt the quaternary structure of non-crosslinked collagen in-vitro.

However, as dentinal collagen is naturally crosslinked, dentinal collagen should be more chemically stable and more resilient to chemical assaults by free-radical byproducts. In order to test this, demineralised dentinal collagen was also exposed directly to CP gel (either 5\% or 10\%) for 4 hours. Figure 3-b-i) presents a mesio-distal cross-section of a demineralised dentine surface with an area exposed to a $10 \% \mathrm{CP}$ droplet. The dentinal collagen not exposed to the CP holds its native fibrillary form (Figure 3-b-ii), organised concentrically around the dentinal tubules. However, the collagen affected by the CP displays the same glassy amorphous layer appearance (Figure 3-b-iv) as found in the collagen scaffold, illustrating that even crosslinked collagen can be affected structurally by CP. Similar to what was observed for the collagen scaffold model, a front of degradation can be observed beyond the edge of the CP droplet as displayed in Figure 3-b-iii). It is interesting to note that Jiang et al (Jiang et al. 2018), suggested that HP would bleach normal dentin mainly by oxidising dentine phosphoproteins (DPP) which are the main non-collagenous protein in dentine. As such it is likely that HP would also affect collagen as reported elsewhere (Jiang et al. 2007; Lopes et al. 2018; Plotino et al. 2008) and is confirmed in our findings. Exposed or non-mineralised collagen found within native dentinal tubules could be directly or indirectly affected by the use of CP as part of the tooth whitening process. However, inter-tubular dentine is mineralised and the impact of CP on mineralised dentinal collagen can be observed spectroscopically by Raman spectroscopy. 


\section{- Evaluation of the impact of CP on collagen in mineralized dentine}

As demonstrated previously (Bakker Schut et al. 2000; Chun-Te Ko et al. 2005; Ramakrishnaiah et al. 2015), Raman spectroscopy can be used as a non-invasive method to detect the chemical composition of mineralised dentine. In our study, we used this approach to monitor the impact of $\mathrm{CP}$ on the chemical fingerprint of mineralised dentine. Figure 4-a shows the Raman spectrum of a control dentine sample with the following four Raman bands of interest for our study: strong phosphate band $v 1$ at $960 \mathrm{~cm}^{-1}$; strong carbonate peak $v 1$ at $1072 \mathrm{~cm}^{-1}$; amide III band doublet at 1245 and $1268 \mathrm{~cm}^{-1}$ and amide I band at $1640 \mathrm{~cm}^{-1}$ (Tsuda et al. 1996). Figures 4-b \& c present the integrated areas of the amide I and III bands respectively at the specific locations across the buccal-lingual axis on control dentine crosssectioned samples. There was no significant difference $(p>0.05)$ of the dentine organic components (amide I and amide III) for the controls at the different locations. These measurements were repeated on the test samples exposed to either $5 \%$ or $10 \% \mathrm{CP}$ concentration for 2 or 4 hours (Figure 5). The Raman spectra showed a significant decrease $(p \leq 0.01)$ in the integrated area of amide I and amide III values in the four test groups (Figure 5-a \& b). In all groups, the measurement location nearest to the site of CP exposure (Loc1) was the most affected (decrease in both amide I and III), whilst the measurement furthest from exposure site was the least affected (Loc4). These results support the fact that peroxide and free-radicals are able to diffuse through the dentine from one exposure window. In addition, the amide $I$ is progressively affected as both the exposure time and concentration is increased. In comparison, the effect on amide III is similar for all the test groups except $(5 \% \mathrm{CP} / 2 \mathrm{hrs})$ $\mathrm{CP}$ exposure. Previous analyses of the Raman spectroscopy of collagen have assigned the amide III vibrations to two different secondary structures: random coil 
and alpha or triple helix (Bonifacio and Sergo 2010; Dehring et al. 2006; Wisniewski et al. 2007) . Thus, a reduction in the overall intensity of the amide III as a function of CP exposure would imply a reduction in the amount of collagen triple helices, suggesting collagen denaturation. The amide I band, however, is associated with protein contents in dentine or bone, and its reduction would imply that dentinal proteins (not just collagen) are being affected by the whitening protocol, as previously discussed. Spectra obtained in this study suggests that CP causes a change in dentine structure (Chng et al. 2005; Kawamoto and Tsujimoto 2004; Rotstein et al. 1996). This reduction in the amide I \& III bands can be attributed to the oxidative and acidic nature of CP (Basting et al. 2003; Joiner 2006) possibly supplemented by further collagen degradation through activation of dentinal MMPs in-vivo (Sato et al. 2013; Toledano et al. 2011)

Whilst it is not possible to physically "see" the structural impact of CP onto collagen using Raman Spectroscopy, the clear reduction in the Amide I and III band intensities are clear indicators that collagen is affected by this whitening protocol. This has to be a concerning outcome, especially as there are no mechanisms for collagen to rejuvenate in the dentine, besides via the formation of secondary and tertiary dentine. The denaturation process of collagen is irreversible and would impact the tooth overall mechanical stability. Collagen in dentine has the primary role of providing an elastic scaffold to support the harder mineralised hydroxy-apatite. The loss or reduction in the collagen content would impact the elastic response of the dentine, by rendering it more brittle. This may result in a greater prominence of tooth cracking for example. As a result, the long term effect of a regular use of such whitening peroxide based products need to be carefully reviewed and end-user need to be aware of its potential impacts. 
Based on the outcomes of this study, the null hypothesis is rejected and concludes that collagen is structurally affected by the application of CP at either $5 \%$ or $10 \%$ invitro.

\section{Conclusion}

Although tooth whitening kits have become increasingly popular, their impacts on dentinal collagen cannot be underestimated. Despite confirming an improvement in tooth whitening using the $10 \% \mathrm{CP}$, a clear impact on the quaternary structure of the dentinal collagen was recorded for all CP concentrations and application protocols. The clear gelatinisation of the collagen and the net reduction in the Raman band intensities for collagen are clear markers of the deleterious effect of CP on dentine. Within the limitation of this in-vitro study, questions may be raised about the unknown long-term implications and ethicacy of such cosmetic trends upon the long-term prognosis of treated teeth. 


\section{Author Contributions}

O. Redha contributed to conception, design, data acquisition, analysis \& interpretation and drafted the manuscript; A. Strange, A. Maeva, N. Mordan \& R. Sambrook, contributed to the interpretation of the data and critically revised the manuscript. Finally, A. McDonald and L. Bozec contributed to conception, design, data analysis \& interpretation, drafted and critically revised the manuscript.

All authors gave final approval and agree to be accountable for all aspects of the work.

\section{Author disclaimer}

The authors have purposely decided not to disclose the brand or manufacturer of the commercially available products used in this study.

\section{Acknowledgements}

The authors would like to thank and gratefully acknowledge the Kuwait Government through Kuwait Cultural Office in London for supporting the research. The authors declare no potential conflicts of interest with respect to the authorship and/or publication of this article. 


\section{Figure captions:}

\section{Figure 1:}

a) Schematic depicting the four different measurement sites along scalpel reference line perpendicular to enamel window for Raman spectroscopy, b) \& c) Dentine collagen spectra shown in Raman microscopy.

\section{Figure 2:}

Example of spectroshade analysis of teeth samples before and after whitening treatment with $5 \% \mathrm{CP}(\mathrm{a})$ and $10 \% \mathrm{CP}(\mathrm{b})$.

\section{Figure 3:}

a) Schematic of a collagen scaffold with areas non-exposed to CP, exposed to a droplet of $5 \% \mathrm{CP}$ and exposed to $10 \% \mathrm{CP}$. The SEM images inset on i) display the droplet on the gel as well as the front of gelatinisation (denoted by *) spreading away from the droplet. Image ii, iii and iv present higher resolution images of the control scaffold surface (ii), the boundary between the glassy gelatinised area of collagen radiating from the CP droplet $(5 \%)$ and the collagen matrix presenting significant morphological degradation of fibrils (iii), the boundary between the glassy gelatinised area of collagen radiating from the CP droplet (10\%) and the collagen matrix presenting an advanced state of degradation but with collagen fibrils recognisable (iv).

b) Schematic of the tooth cross-section exposed a droplet of CP (10\%). SEM images ii, iii and iv display respectively the dentine area not exposed to CP (ii), in close proximity to the droplet (iii) and immediately in the area where the droplet was deposited (iv).

Figure 4:

Reference Raman Spectrum of control dentine sample (a) and box plots of the integrated area of the amide I (b) and amide III (c) obtained from spectra recorded at the 4 locations of interest along the mesial-buccal axis. Note that Loc1: $1 \mathrm{~mm}$ away from the buccal DEJ, Loc2: $1 \mathrm{~mm}$ away from the buccal pulp, Loc3: $1 \mathrm{~mm}$ away from the lingual pulp wall and Loc4: $1 \mathrm{~mm}$ away from the lingual DEJ.

\section{Figure 5:}

Box plots of the integrated area of the amide I (b) and amide III (c) obtained from spectra recorded at the 4 locations of interest along the mesial-buccal a axis on the exposed dentine to the various whitening protocols $(5 \% \mathrm{CP} / 2 \mathrm{hrs}, 5 \% \mathrm{CP} / 4 \mathrm{hrs}$, $10 \% \mathrm{CP} / 2 \mathrm{hrs}$ and $10 \% \mathrm{CP} / 4 \mathrm{hrs}$ ). Note that Loc1: $1 \mathrm{~mm}$ away from the buccal DEJ, Loc2: $1 \mathrm{~mm}$ away from the buccal pulp, Loc3: $1 \mathrm{~mm}$ away from the lingual pulp wall and Loc4: $1 \mathrm{~mm}$ away from the lingual DEJ. 


\section{$\underline{\text { References }}$}

Bakker Schut T, Witjes M, Sterenborg H, Speelman O, Roodenburg J, Marple E, Bruining $H$, Puppels G. 2000. In vivo detection of dysplastic tissue by raman spectroscopy. Analytical chemistry. 72(24):6010-6018.

Basting RT, RODRIGUES JR AL, Serra MC. 2003. The effects of seven carbamide peroxide bleaching agents on enamel microhardness over time. The Journal of the American Dental Association. 134(10):1335-1342.

Bonifacio A, Sergo V. 2010. Effects of sample orientation in raman microspectroscopy of collagen fibers and their impact on the interpretation of the amide iii band. Vibrational Spectroscopy. 53(2):314-317.

Bozec L, Odlyha M. 2011. Thermal denaturation studies of collagen by microthermal analysis and atomic force microscopy. Biophysical journal. 101(1):228-236.

Cheema U, Brown RA. 2013. Rapid fabrication of living tissue models by collagen plastic compression: Understanding three-dimensional cell matrix repair in vitro. Adv Wound Care (New Rochelle). 2(4):176-184.

Chng H, Ramli H, Yap A, Lim C. 2005. Effect of hydrogen peroxide on intertubular dentine. Journal of Dentistry. 33(5):363-369.

Chun-Te Ko A, Hewko MD, Leonardi L, Sowa MG, Dong CC, Williams P, Cleghorn B. 2005. Ex vivo detection and characterization of early dental caries by optical coherence tomography and raman spectroscopy. Journal of biomedical optics. 10(3):031118.

De Geus J, Wambier L, Boing T, Loguercio A, Reis A. 2018. At-home bleaching with $10 \%$ vs more concentrated carbamide peroxide gels: A systematic review and meta-analysis. Operative dentistry. 43(4):E210-E222.

de l'Eclairage $\mathrm{Cl}$. 1978. Recommendations on uniform color spaces, color-difference equations, psychometric color terms. Paris: CIE.

Dehring KA, Crane NJ, Smukler AR, McHugh JB, Roessler BJ, Morris MD. 2006. Identifying chemical changes in subchondral bone taken from murine knee joints using raman spectroscopy. Applied spectroscopy. 60(10):1134-1141.

Dwinelle W. 1850. Ninth annual meeting of american society of dental surgeons: Article x. Am J Dent Sci. 1:57-61.

Elfallah HM, Bertassoni LE, Charadram N, Rathsam C, Swain MV. 2015. Effect of tooth bleaching agents on protein content and mechanical properties of dental enamel. Acta biomaterialia. 20:120-128.

Goldberg M, Kulkarni AB, Young M, Boskey A. 2011. Dentin: Structure, composition and mineralization: The role of dentin ecm in dentin formation and mineralization. Frontiers in bioscience (Elite edition). 3:711.

Han M, Li Q-L, Cao Y, Fang H, Xia R, Zhang Z-H. 2017. In vivo remineralization of dentin using an agarose hydrogel biomimetic mineralization system. Scientific reports. 7:41955.

Hanks C, Fat J, Wataha J, Corcoran J. 1993. Cytotoxicity and dentin permeability of carbamide peroxide and hydrogen peroxide vital bleaching materials, in vitro. Journal of Dental Research. 72(5):931-938.

Haywood VB. 2006. Nightguard vital bleaching: Indications and limitations. US Dentistry.2-8. Haywood VB, Heymann HO. 1989. Nightguard vital bleaching. Quintessence Int. 20(3):173176. 
Jiang T, Guo Y, Feng X, Sa Y, Yang X, Wang M, Li P, Wang Y. 2018. Hydrogen peroxide might bleach natural dentin by oxidizing phosphoprotein. Journal of Dental Research. 97(12):1339-1345.

Jiang T, Ma X, Wang Y, Zhu Z, Tong H, Hu J. 2007. Effects of hydrogen peroxide on human dentin structure. Journal of dental research. 86(11):1040-1045.

Joiner A. 2006. The bleaching of teeth: A review of the literature. Journal of dentistry. 34(7):412-419.

Kawamoto K, Tsujimoto Y. 2004. Effects of the hydroxyl radical and hydrogen peroxide on tooth bleaching. Journal of Endodontics. 30(1):45-50.

Kou F, Takahama U. 1995. Hydrogen peroxide-induced luminescence and evolution of molecular oxygen in human saliva. Archives of oral biology. 40(1):15-21.

Kwon SR, Wertz PW. 2015. Review of the mechanism of tooth whitening. Journal of Esthetic and Restorative Dentistry. 27(5):240-257.

Leonard RH, Sharma A, Haywood vB. 1998. Use of different concentrations of carbamide peroxide for bleaching teeth: An in vitro study. Quintessence International. 29(8).

Lima SNL, Ribeiro IS, Grisotto MA, Fernandes ES, Hass V, de Jesus Tavarez RR, Pinto SCS, Lima DM, Loguercio AD, Bandeca MC. 2018. Evaluation of several clinical parameters after bleaching with hydrogen peroxide at different concentrations: A randomized clinical trial. Journal of dentistry. 68:91-97.

Lopes FC, Roperto R, Akkus A, Akkus O, Palma-Dibb RG, de Sousa-Neto MD. 2018. Effect of laser activated bleaching on the chemical stability and morphology of intracoronal dentin. Archives of oral biology. 86:40-45.

McKnight-Hanes C, Whitford GM. 1992. Fluoride release from three glass ionomer materials and the effects of varnishing with or without finishing. Caries research. 26(5):345-350.

Meireles S, Heckmann S, Leida F, Santos I, Bona Á, Demarco F. 2008. Efficacy and safety of $10 \%$ and $16 \%$ carbamide peroxide tooth-whitening gels: A randomized clinical trial. Operative Dentistry. 33(6):606-612.

Neel EAA, Bozec L, Knowles JC, Syed O, Mudera V, Day R, Hyun JK. 2013. Collagen-emerging collagen based therapies hit the patient. Advanced drug delivery reviews. 65(4):429456.

Plotino G, Buono L, Grande NM, Pameijer CH, Somma F. 2008. Nonvital tooth bleaching: A review of the literature and clinical procedures. Journal of endodontics. 34(4):394407.

Ramakrishnaiah R, Rehman Gu, Basavarajappa S, Al Khuraif AA, Durgesh B, Khan AS, Rehman lu. 2015. Applications of raman spectroscopy in dentistry: Analysis of tooth structure. Applied Spectroscopy Reviews. 50(4):332-350.

Rao R, Rathod T, Sekhar V, Ch KK. 2016. Comparative evaluation of the bleaching efficacy of $16 \%$ carbamide peroxide and $30 \%$ hydrogen peroxide-an in-vitro study. Indian Journal of Mednodent and Allied Sciences. 4(2).

Rotstein I, Dankner E, Goldman A, Heling I, Stabholz A, Zalkind M. 1996. Histochemical analysis of dental hard tissues following bleaching. Journal of Endodontics. 22(1):23-26.

Sato C, Rodrigues FA, Garcia DM, Vidal CM, Pashley DH, Tjaderhane L, Carrilho MR, Nascimento FD, Tersariol IL. 2013. Tooth bleaching increases dentinal protease activity. J Dent Res. 92(2):187-192.

Soares DG, Basso FG, Hebling J, de Souza Costa CA. 2014. Concentrations of and application protocols for hydrogen peroxide bleaching gels: Effects on pulp cell viability and whitening efficacy. J Dent. 42(2):185-198. 
Sulieman M, MacDonald E, Rees JS, Newcombe RG, Addy M. 2006. Tooth bleaching by different concentrations of carbamide peroxide and hydrogen peroxide whitening strips: An in vitro study. J Esthet Restor Dent. 18(2):93-100; discussion 101.

Toledano M, Yamauti M, Osorio E, Osorio R. 2011. Bleaching agents increase metalloproteinases-mediated collagen degradation in dentin. J Endod. 37(12):16681672.

Tsuda H, Ruben J, Arends J. 1996. Raman spectra of human dentin mineral. European journal of oral sciences. 104(2):123-131.

Walsh $\amalg .2000$. Safety issues relating to the use of hydrogen peroxide in dentistry. Australian dental journal. 45(4):257-269.

Wisniewski M, Sionkowska A, Kaczmarek H, Lazare S, Tokarev V, Belin C. 2007. Spectroscopic study of a krf excimer laser treated surface of the thin collagen films. Journal of Photochemistry and Photobiology A: Chemistry. 188(2-3):192-199. 
Figure 1

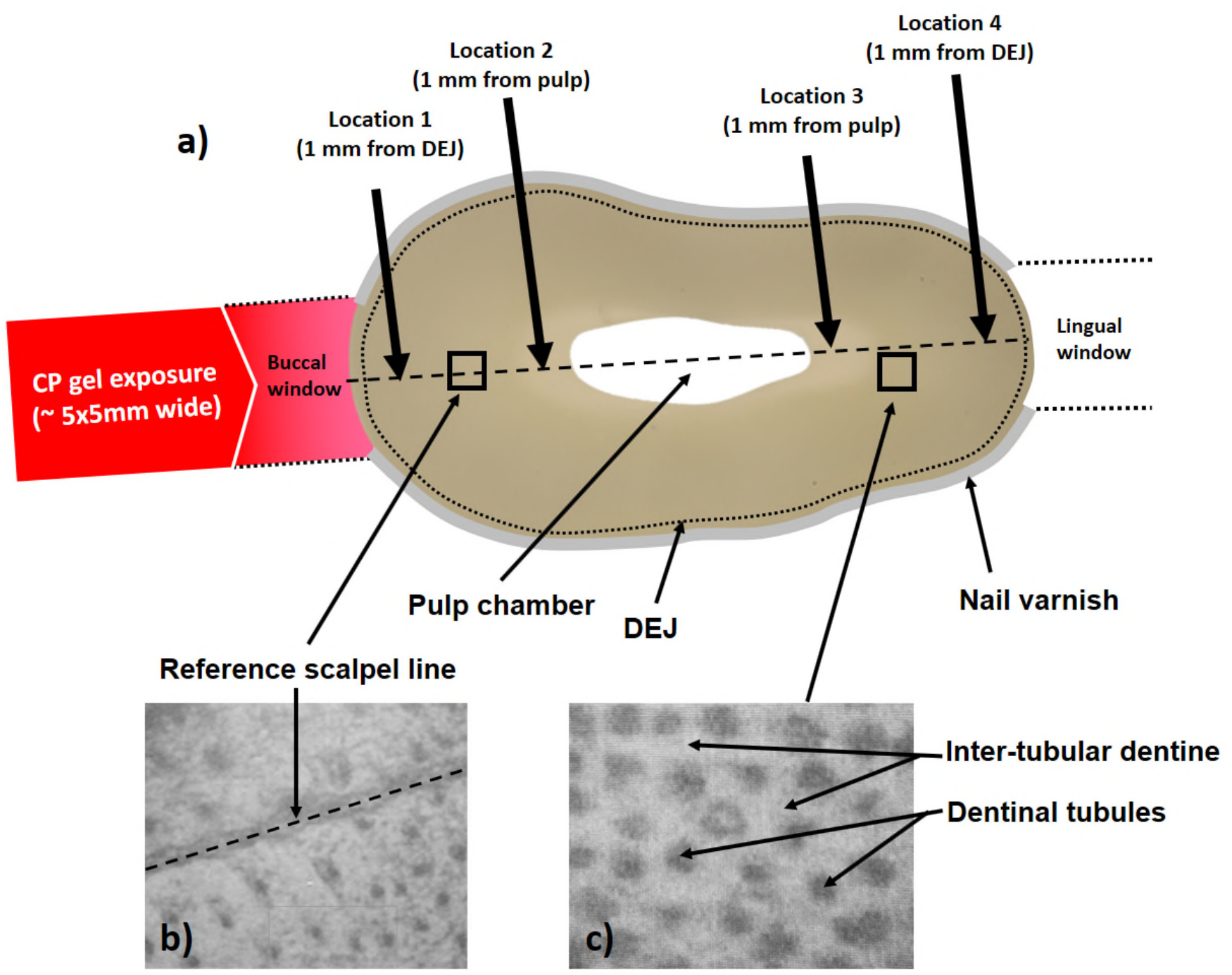


Figure 2

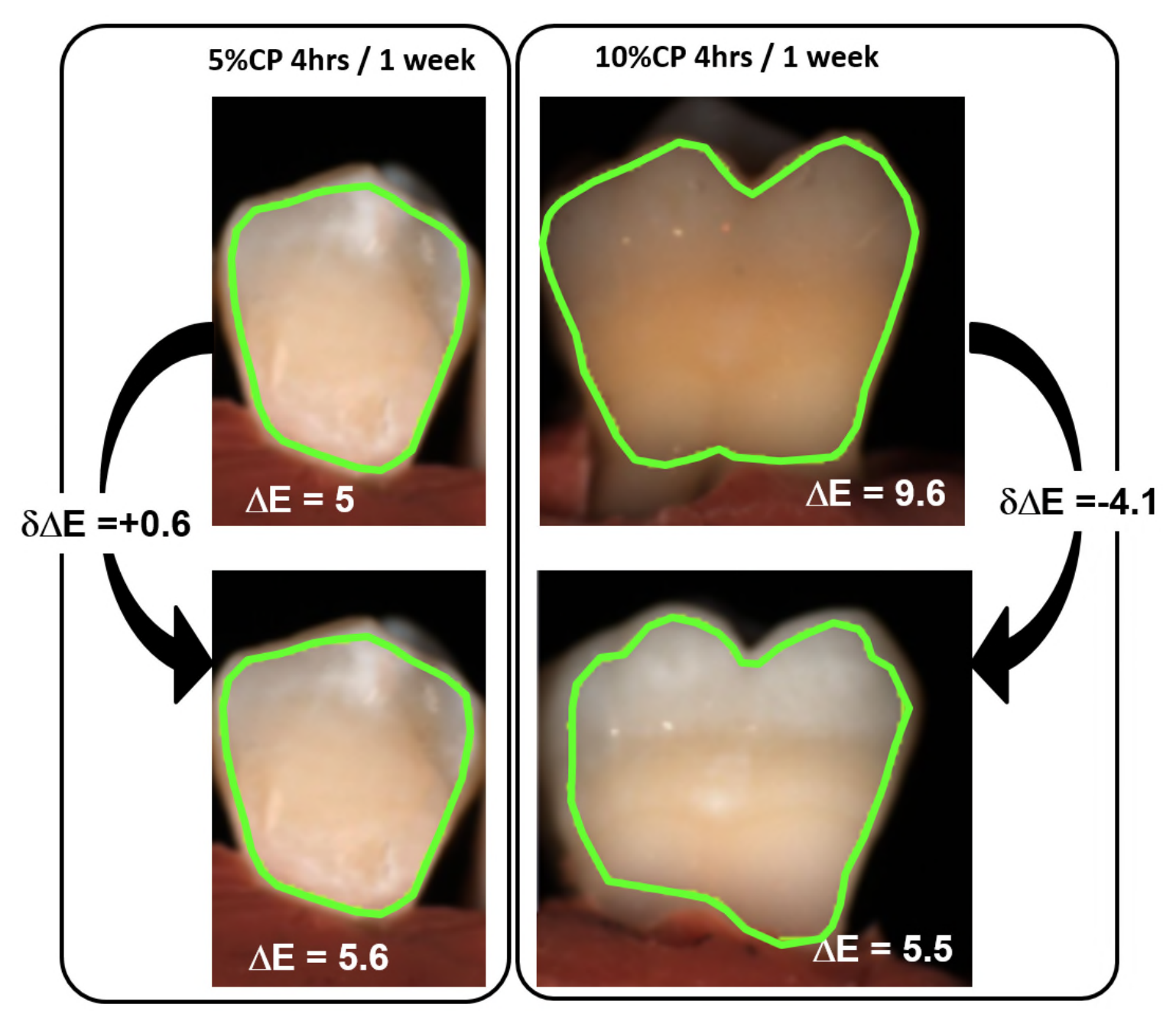


Figure 3

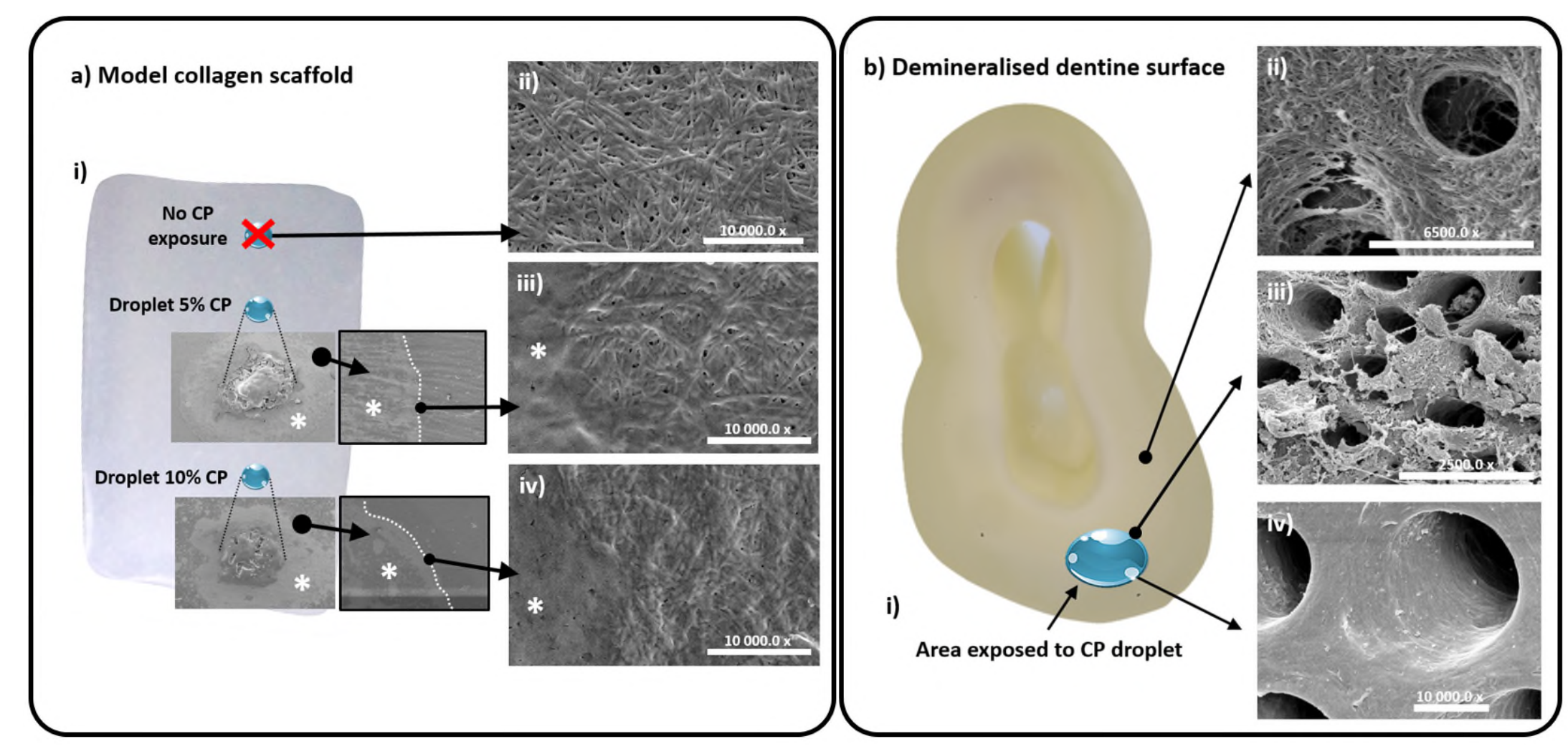


Figure 4

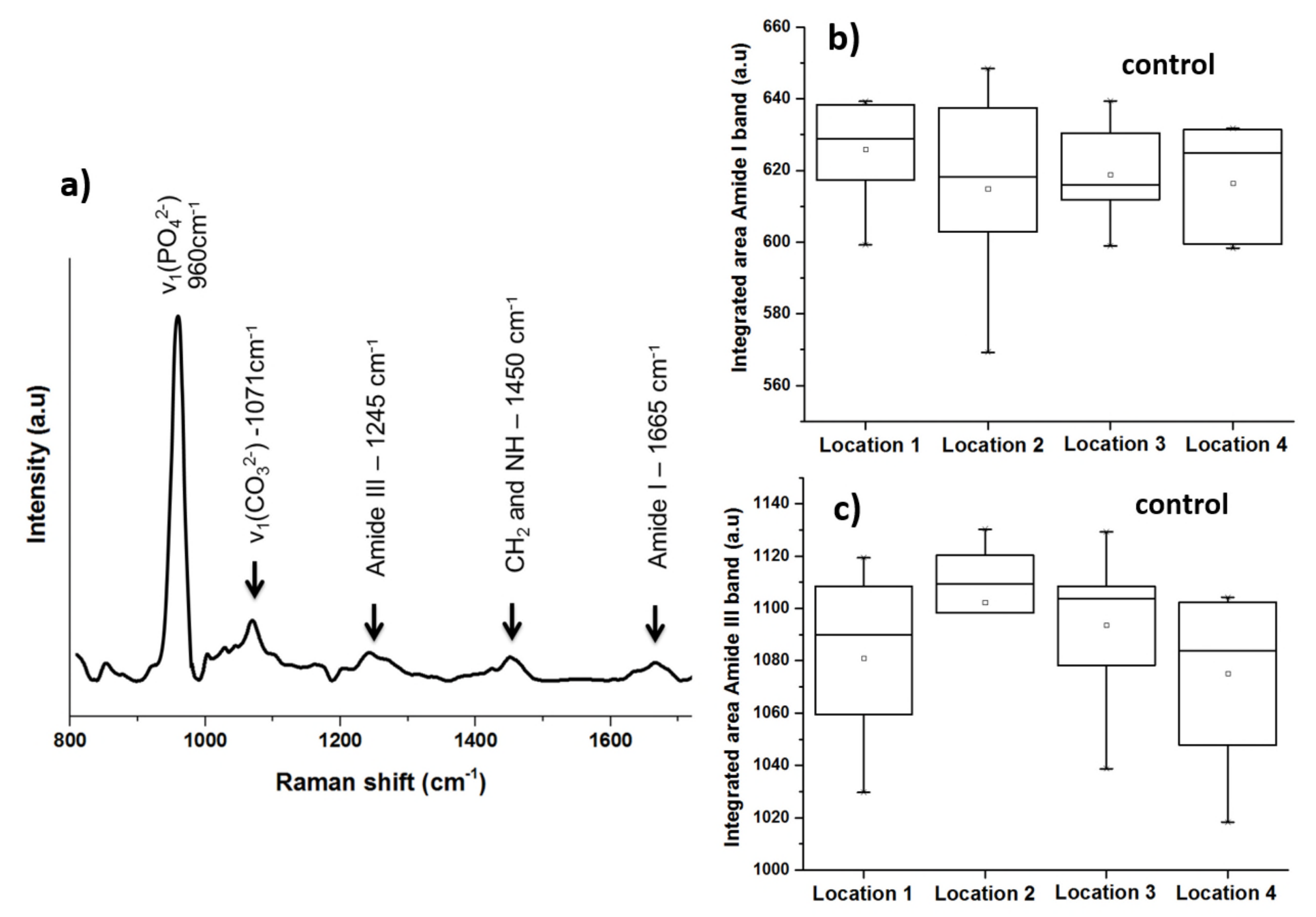


Figure 5

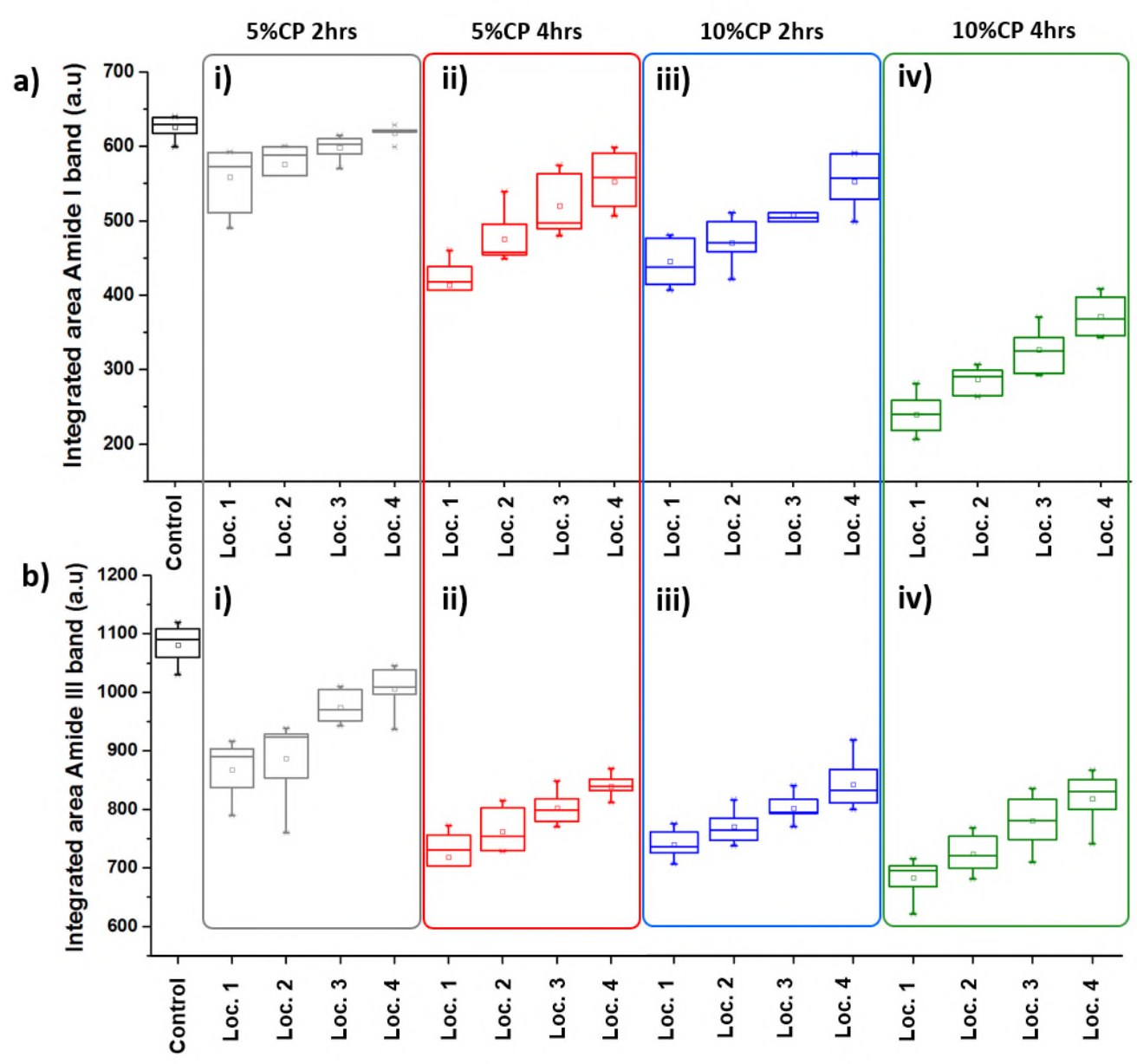

